\title{
Links between conscious awareness and response inhibition: Evidence from masked priming
}

\author{
MARTIN EIMER \\ Birkbeck College, University of London, England \\ and \\ FRIEDERIKE SCHLAGHECKEN \\ University of Warwick, Coventry, England
}

\begin{abstract}
Recent results from "subliminal priming" experiments have shown that masked prime stimuli which can not be consciously perceived can trigger response activation processes, but that these response activations can later be subject to inhibition. Links between conscious awareness and response inhibition were investigatedby manipulating the visibility of masked prime stimuli, from clearly visible primes to prime stimuli that were inaccessible to conscious perception. Response inhibition was observed with unperceived prime stimuli, but not for suprathreshold primes. Correlations between individual prime identification thresholds and the onset of response inhibition indicate that the absence or presence of conscious awareness can predict whether or not response inhibition is elicited. These results demonstrate qualitative differences in the effects of conscious and unconscious information. It is argued that response facilitation produced by consciously available perceptual information can counteract automatic effects of self-inhibitory motor control circuits.
\end{abstract}

Few issues in psychological research are more controversial than the status of conscious awareness and the question of how consciousness affects cognitive processes and the control of voluntary action. Can behavior be influenced by sensory information that is not consciously accessible? Are such "subliminal" effects qualitatively different from the effects of consciously perceived (supraliminal) stimuli?

A number of recent studies have shown that response tendencies can be activated by stimuli presented below the threshold of conscious awareness (e.g., Dehaene et al., 1999; Eimer, 1999; Eimer \& Schlaghecken, 1998; Leuthold \& Kopp, 1998; Neumann \& Klotz, 1994; Schlaghecken \& Eimer, 2000, 2002). In these "subliminal priming" experiments, masked prime stimuli were presented prior to a response-relevant target. Primes and targets were mapped to the same response in compatible trials, and to different responses in incompatible trials. Although participants were unable to identify the masked primes, responses to the clearly visible targets were affected by prime-target compatibility. When primes and targets were presented in close temporal succession, responses in compatible trials were faster and more accurate than responses in incompatible trials ("positive compatibility effect"). Electro-

The present research was supported by a grant from the Biotechnology and Biological Sciences Research Council (BBSRC). The authors thank Phillip Merikle and two anonymous reviewers for constructive comments. Correspondence should be addressed to M. Eimer, Department of Psychology, Birkbeck College, University of London, Malet Street, London WC1E 7HX, England (e-mail: m.eimer@bbk.ac.uk). physiologicalstudies which measured the lateralized readiness potential (LRP) as an index of selective response preparation and activation (see Eimer, 1998, for details on the derivation and interpretation of the LRP) found that the masked primes triggered a partial activation of their corresponding response (Dehaene et al., 1999; Eimer, 1999; Eimer \& Schlaghecken, 1998; Leuthold \& Kopp, 1998). These findings suggest that response processes are influenced by direct perceptuomotor links (Neumann, 1990), which allow sensory information access to motor stages, even when a subsequent mask makes this information inaccessible to conscious awareness.

However, subliminal priming does not always result in an advantage for compatible trials. Performance benefits for compatible trials observed with short prime-target intervals turn into performance costs when these intervals are increased (Eimer, 1999; Eimer \& Schlaghecken, 1998; Schlaghecken \& Eimer, 1997, 2000). The surprising and almost paradoxical observation that performance is better on incompatible than on compatible trials ("negative compatibility effect") has been linked to an inhibition of the response tendency initially triggered by the prime. In line with this hypothesis, recent LRP studies (Eimer, 1999; Eimer \& Schlaghecken, 1998) have found that an early partial activation of the response assigned to the prime is later replaced by an LRP of opposite polarity, as would be expected if the initially activated response was subject to inhibition.

Although these experiments have revealed the existence of an inhibitory component in subliminal priming, the link 
between consciousness and response inhibition has not yet been studied directly. Because masked prime stimuli have always been inaccessible to conscious awareness in previous studies, it is unclear whether qualitatively different effects might have been observed with suprathreshold primes. We assume that the sequence of "facilitation followed by inhibition" observed in subliminal priming reflects a basic principle in motor control. Response activation and inhibition act as opponent processes, with inhibition as an automatic consequence of response activation. One likely function of such self-inhibitory control processes is the maintenance of stability within the motor system. Selfinhibition thus shuts down response tendencies unless their activation level is sustained by facilitatory input from other sources (see Houghton \& Tipper, 1994, for a functional model of self-inhibition in the control of response processes). One primary source of response facilitation is perceptual evidence indicating that a currently activated response is appropriate. When such evidence is available, response facilitation will dominate inhibition. Under subliminal priming conditions, however, the mask eliminates sensory representations of the prime stimulus. Because automatic self-inhibition is no longer counteracted by facilitatory perceptual input, the initial response activation will be subject to inhibition (see Schlaghecken \& Eimer, 2002, for a more detailed account).

Because a removal of sensory representations of a prime by a subsequently presented masking stimulus makes the prime inaccessible to conscious awareness, the presence or absence of response inhibition and the presence or absence of conscious awareness could be consequences of the same process. If that was the case, response inhibition and conscious awareness should be correlated. Inhibition should be found when masked primes cannot be consciously perceived, but not when they remain above the threshold of conscious awareness. By measuring whether a stimulus can be consciously discriminated, we should be able to predict whether response tendencies triggered by this stimulus will or will not be subject to inhibition.

The aim of the present study was to test this hypothesis that conscious awareness and response inhibition are correlated: Primed response tendencies are subject to inhibition when primes are inaccessible to awareness, but not when they are consciously perceived. To obtain experimental evidence for these assumptions, we manipulated the visibility of masked prime stimuli to create a "prime visibility gradient," ranging from clearly suprathreshold primes to subthreshold prime stimuli. The masking stimuli consisted of randomly arranged and partially overlapping line elements (see Figure 1). In Experiment 1, a prime visibility gradient was generated by varying the number of line elements constituting a mask between 0 (no mask) and 30 (dense mask), while prime duration remained constant $(16 \mathrm{msec})$. In Experiment 2, we varied prime duration between 96 and $16 \mathrm{msec}$, while mask density remained constant (30 elements). Prime-target interval was $150 \mathrm{msec}$, which is sufficiently long to result in negative compatibility effects when primes are fully masked (Schlaghecken
\& Eimer, 2000). Trials were compatible or incompatible, and the presence or absence of response inhibition for each prime visibility condition was inferred from performance differences between compatible and incompatible trials. If response inhibition and the absence of conscious awareness are correlated, the point along the visibility gradient where primes become inaccessible to conscious awareness should coincide with the point along this gradient where response inhibition starts. Because observers will differ considerably in their ability to perceive masked primes when their visibility is reduced, individual perception thresholds should predict the onset of response inhibition for individual participants.

To demonstrate this relationship, prime identification thresholds and the onset of response inhibition need to be determined independently. We measured individual identification thresholds at the beginning of each experiment by using an adaptive weighted up-down psychophysical staircase (Kaernbach, 1991). Starting with prime stimuli that were unmasked (Experiment 1) or presented for $96 \mathrm{msec}$ (Experiment 2), prime visibility was decreased whenever primes were correctly identified. Identification performance was expected to reach chance level at some point along the prime visibility gradient. We measured the onset of response inhibition in subsequent blocks in which masked primes were followed by response-relevant targets and prime visibility was varied between blocks. We expected to find systematic qualitative differences in the impact of suprathreshold and subthreshold primes on behavior: The onset of response inhibition was defined as the point along the prime visibility gradient where primetarget compatibility effects turn from positive (suprathreshold primes, no response inhibition) to negative (subthreshold primes, response inhibition). This "turning point" was determined individually for each participant (see the Method section for details).

If the presence or absence of conscious awareness and the presence or absence of response inhibition result from the same underlying process, identification performance (defining the individual threshold of conscious awareness) and the onset of response inhibition should be correlated: For participants showing good prime identification performance, response inhibition should be found only when prime visibility is low. In contrast, for participants with poor identification performance, response inhibition should already be observed earlier along the prime visibility gradient.

\section{EXPERIMENT 1}

\section{Method}

Participants. Twelve paid volunteers (6 male), 19-42 years of age $($ mean $=26.3$ years $)$, participated in the experiment. Three other participants had to be excluded because of an overall error rate of about $10 \%$ or more. According to self-report, all participants but 1 were right-handed, and all had normal or corrected-to-normal vision.

Stimuli and Apparatus. Left-pointing and right-pointing arrows $(<<,>>)$ served as primes and targets, subtending a visual angle of approximately $0.9^{\circ} \times 0.3^{\circ}$. Masking stimuli were constructed 
from a $6 \times 5$ matrix, randomly filled with overlapping horizontal, vertical, and oblique lines of different lengths $\left(0.06^{\circ}\right.$ to $\left.0.3^{\circ}\right)$, resulting in a roughly rectangular array of about $1.4^{\circ} \times 0.9^{\circ}$. Line width was identical for all stimuli $\left(0.06^{\circ}\right)$.

Procedure. The participants were seated in a dimly lit chamber with response keys under their left and right index fingers. A computer screen was placed $100 \mathrm{~cm}$ in front of the participants' eyes so that the screen center was in the center of the participants' horizontal straight-ahead line of sight. The participants were instructed to maintain central eye fixation. The experiment consisted of a staircase block of 248 trials, two practice blocks (20 trials each), and 21 masked priming blocks ( 80 trials each).

In the masked priming blocks, each trial consisted of a prime stimulus (16-msec duration), immediately followed by a mask (100msec duration), followed by a $50-\mathrm{msec}$ blank screen, which in turn was followed by a target stimulus (100-msec duration). The intertrial interval (ITI) was 1,300 msec (see Figure 1, left). Trials were termed compatible when prime and target arrows were pointing in the same direction, and incompatible otherwise. Both conditions were equiprobable, randomized within each block. The participants were instructed to respond as fast and as accurately as possible with a left keypress to left-pointing target arrows and with a right keypress to right-pointing target arrows. Seven different mask densities were employed: 0 (blank screen), 5, 10, 15, 20, 25, and 30 elements. Mask density varied between blocks, and each of the seven mask density conditions (mask0 to mask30) was delivered in a series of three consecutive blocks. The order of these seven series was randomized for each participant.

At the beginning of the experiment, a staircase block was run, consisting of two series of 124 trials, interrupted by a break. In this block, primes (i.e., arrow stimuli) and masks were presented as in the masked priming blocks, but no target followed the mask. The ITI was $2,000 \mathrm{msec}$, and the mask density was varied. Each of the two series started with a 0-element mask (i.e., an unmasked arrow). The participants had to identify the direction of the arrow and to respond with the corresponding key. A fixed-step, weighted 1 up/2 down procedure was employed: After a correct response, the mask density on the next trial was increased by 1 element; after an incorrect response, it was decreased by 2 elements, within the range of $0-30$ elements. With this procedure, identification performance is assumed to converge on a $66 \%$ correct level (Kaernbach, 1991). ${ }^{1}$ The participants were told that this would result in a subjectively near chance performance level. They were encouraged to respond on each trial even when they were unsure about the direction of the arrow.

Data analysis. Repeated measures analyses of variance (ANOVAs) were computed on correct reaction times (RTs) and error rates in the masked priming blocks for the factors mask density (mask0, mask5, mask10, mask15, mask20, mask25, and mask30) and compatibility (compatible, incompatible). Where appropriate, GreenhouseGeisser adjustments to the degrees of freedom were performed (indicated in the Results section by $\varepsilon$ ). The onset of response inhibition was estimated by first computing prime-target compatibility effects (RT on incompatible trials minus RT on compatible trials) for each mask density condition and then fitting a regression line between the last positive compatibility effect larger than $5 \mathrm{msec}$ and the first negative compatibility effect larger than $5 \mathrm{msec}$. The number of mask elements corresponding to the zero crossing point of this regression line defined the onset of response inhibition along the prime visibility gradient.

From the staircase data, the first 30 trials of each series were discarded as warm-up trials. From the remaining $2 * 94$ trials, mean mask density was computed for correct-incorrect reversals (first incorrect response after one or more correct responses) and for incorrect-correct reversals (first correct response after one or more incorrect responses). The mean of these two values served as the identification threshold estimate. Correlations were computed between individual identification threshold and individual response inhibition onset estimates.

\section{Results}

Reaction times were longer in the mask 0 condition than in the other conditions, resulting in a main effect of mask on RTs $[F(6,66)>4.87, p<.007, \varepsilon=.489]$. Error rates tended to be lower in the mask 0 condition than in the other

\section{Experiment 1: Different Mask Densities}

\section{Experiment 2: Different Prime Durations}

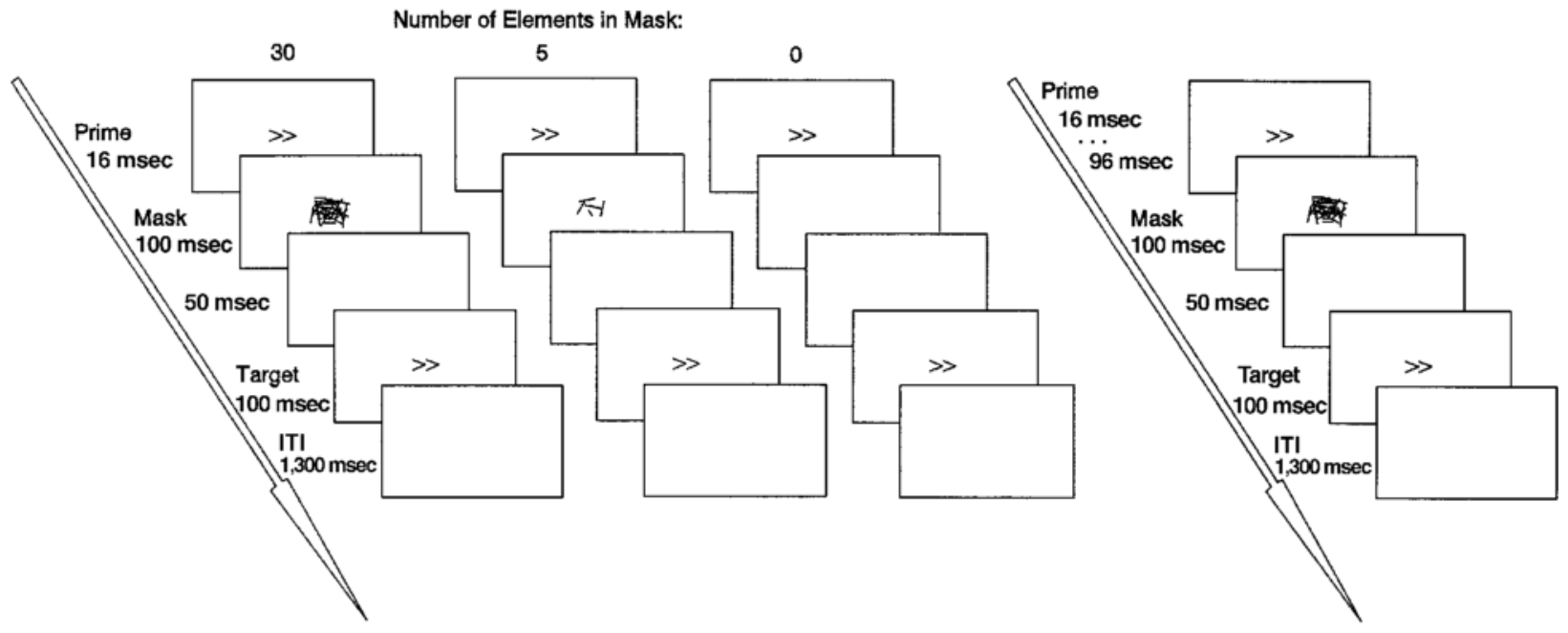

Figure 1. Illustration of stimulus configuration and trial structure in Experiments 1 and 2. Left panel: Compatible trials in Experiment 1 in the mask30, mask5, and mask0 conditions. Right panel: Compatible trials in Experiment 2, in which prime duration was varied between 16 and $96 \mathrm{msec}$. 
conditions, but this difference was not significant $[F(6,66)<$ $2.2, p>.088]$. There was no main effect of compatibility on either RTs or error rates [both $F_{\mathrm{s}}(1,9)<2.1$, both $p_{\mathrm{s}}>$ .18]. As can be seen from Figure 2 (top panel), RTs were faster and fewer errors were made on compatible than on incompatible trials in the mask 0 and mask 5 conditions, whereas the reverse was true for the remaining conditions [for the interactions of compatibility $\times$ mask on RTs and error rates, both $F_{\mathrm{s}}(6,56)>15.0$, both $p_{\mathrm{s}}<.001, \varepsilon=.283$ and .542 , respectively]. Paired $t$ tests, comparing RTs (error rates) on compatible trials with RTs (error rates) on incompatible trials, separately for each masking condition, confirmed the existence of positive compatibility effects in the mask0 condition [both $t \mathrm{~s}(11)>5.0$, both $p \mathrm{~s}<$ $.001]$. In the mask 5 condition, a numerical positive compatibility failed to reach significance [both $t \mathrm{~s}(11)<1.6$, both $p \mathrm{~s}>$.14]. With all masks containing 10 or more elements, negative compatibility effects were present for RTs [all $t \mathrm{~s}(12)>3.69$, all $p \mathrm{~s}<.004$ ] as well as error rates [all $t \mathrm{~s}(12)>2.52$, all $p \mathrm{~s}<.029$. $^{2}$

The mean identification threshold measured in the staircase block corresponded to a mask density of 7.9 elements. The estimated onset of response inhibition (averaged across participants) corresponded to a mask density of 6.8 elements. As predicted, a significant positive correlation between these two estimates was obtained ( $r=$ $.63, p<.027$, two-tailed). Figure 2 (bottom panel), where individual identification threshold estimates are plotted against inhibition onset estimates, shows that participants with good identification performance tended to show response inhibition only with dense masks. For participants with poor identification performance, response inhibition tended to be elicited earlier along the prime visibility gradient. However, Figure 2 also reveals that although significant, this correlation pattern was by no means perfect. This could be related to the fact that different step sizes were used to determine detection threshold and inhibition onset estimates. Whereas mask density was varied in steps of 5 mask elements in the masked priming blocks, step sizes of 1 element were used in the staircase blocks. This problem was eliminated in Experiment 2.

\section{EXPERIMENT 2}

\section{Method}

Participants. Twelve paid volunteers (3 male), 19-37 years of age $($ mean $=26.6$ years) participated in the experiment. According to self-report, all participants but one were right-handed, and had normal or corrected-to-normal vision.

Stimuli and Apparatus were identical to those in Experiment 1, but only one type of mask (30 elements) was employed.

Procedure. The experimental set-up was similar to that of Experiment 1 , except that instead of manipulating mask density, we varied prime duration (see Figure 1, right). The experiment consisted of a staircase block of 120 trials, one practice block (20 trials), and 12 masked priming blocks (80 trials each). In the latter blocks, prime duration was blocked and was either 96, 80, 64, 48, 32, or $16 \mathrm{msec}$. Each of these blocks was presented twice, and the sequence of blocks was completely randomized for each participant. Otherwise, the conditions were identical to those in Experiment 1. At the beginning of the experiment, a staircase block was run, consisting of two series of 60 trials, interrupted by a 20 -sec break. The staircase procedure was similar to that in Experiment 1, except that instead of mask density, prime duration was varied. Each staircase series started with a 160 -msec prime duration. After a correct response, prime duration was reduced by $16 \mathrm{msec}$; after an incorrect response, it was prolonged by $32 \mathrm{msec}$, within the range of $16-160 \mathrm{msec}$.

Data analysis. Repeated measures ANOVAs were computed on correct RTs and error rates in the masked priming blocks for the factors prime duration ( prime96, prime80, prime64, prime48, prime 32 , and prime16) and compatibility (compatible, incompatible). From the staircase data, the first 10 trials of each series were discarded. From the remaining $2 * 50$ trials, individual identification thresholds were computed. Identification thresholds, inhibition onset estimates, and correlations between them were computed as in Experiment 1, except that these variables were now defined in terms of prime duration (in milliseconds). One participant (who was among the 3 participants showing the worst prime identification performance in the staircase blocks) produced negative compatibility effects even with the longest prime durations. This participant had to be excluded from the correlation analysis, because no response inhibition onset estimate could be computed.

\section{Results}

RTs and error rates increased with increasing prime duration, resulting in a main effect of duration that was significant for RTs $[F(5,55)=7.25, p<.001, \varepsilon=.751]$ and approached significance for error rates $[F(5,55)=2.65$, $p<.057, \varepsilon=.675]$. Errors were more frequent on incompatible trials than on compatible trials $[F(1,11)=9.5$, $p<.01$ ], while there was no main effect of compatibility on RTs $(F<0.6, p>.47)$. The interaction of compatibility and prime duration was highly significant for both RTs and error rates [both $F \mathrm{~s}(5,55)>7.8$, both $p \mathrm{~s}<.001, \varepsilon=$ .481 and .742, respectively]. As can be seen from Figure 3 (top panel), RTs were faster and fewer errors were made on compatible than on incompatible trials for the prime96, prime 80 , and prime 64 conditions, but the reverse was true for the prime 32 and prime 16 conditions. Paired $t$ tests, comparing RTs (error rates) on compatible trials with RTs (error rates) on incompatible trials for each prime duration condition separately confirmed the existence of positive compatibility effects in the prime 96 and prime 80 conditions on RTs and error rates [all $t \mathrm{~s}(11)>2.7$, all $p s<.017$ ], and the presence of negative compatibility effects in the prime 16 and prime 32 conditions on RTs [both $t \mathrm{~s}(11)>3.6$, both $p \mathrm{~s}<.004]$. No significant compatibility effects were found in the prime 48 and prime 64 conditions [all $t \mathrm{~s}(11)<2.1$, all $p \mathrm{~s}>.068$ ].

The identification threshold (averaged across 11 participants) corresponded to a prime duration of $56 \mathrm{msec}$. The estimated onset of response inhibition (averaged across 11 participants) corresponded to a prime duration of $54 \mathrm{msec}$. As predicted, a highly significant positive correlation between these two estimates was obtained $(r=.743, p<$ .009 , two-tailed). In Figure 3 (bottom panel), individual identification threshold estimates are plotted against inhibition onset estimates. Participants with good identification performance showed evidence for response inhibition only when primes were presented very briefly. For participants with poor identification performance, response inhibition was already present for longer prime durations. 

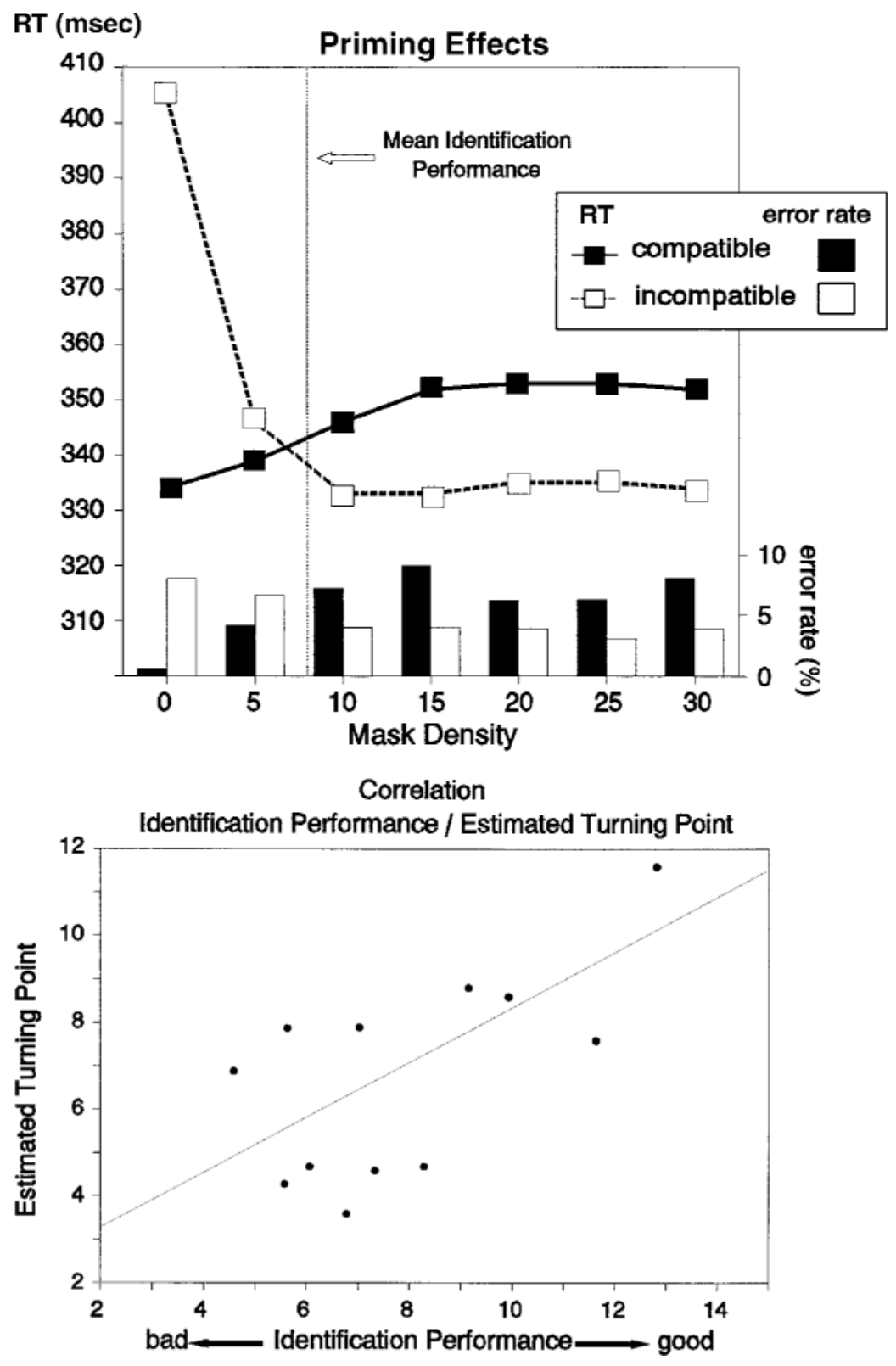

Figure 2. Results of Experiment 1. Top panel: Reaction times (line graphs) and error rates (bar graphs) in compatible trials (black) and incompatible trials (white) for the different mask density conditions ranging from 0 (no mask) to 30 (dense mask). The vertical line indicates the prime identification threshold along the prime visibility gradient as determined with psychophysical staircases. Bottom panel: Individual estimated turning points from positive to negative compatibility effects plotted against individual prime identification thresholds. Both values are defined in terms of mask density (number of mask elements). Low values indicate "early" turning points and poor identification performance; high values indicate "late" turning points and good identification performance.

\section{DISCUSSION}

The aim of this study was to substantiate the claim that conscious awareness and the presence or absence of response inhibition in subliminal priming are correlated.
Two experiments tested the hypothesis that response inhibition is elicited when masked primes become inaccessible to conscious awareness, but not with suprathreshold primes. Prime-target compatibility effects were measured while the visibility of masked prime stimuli was varied 

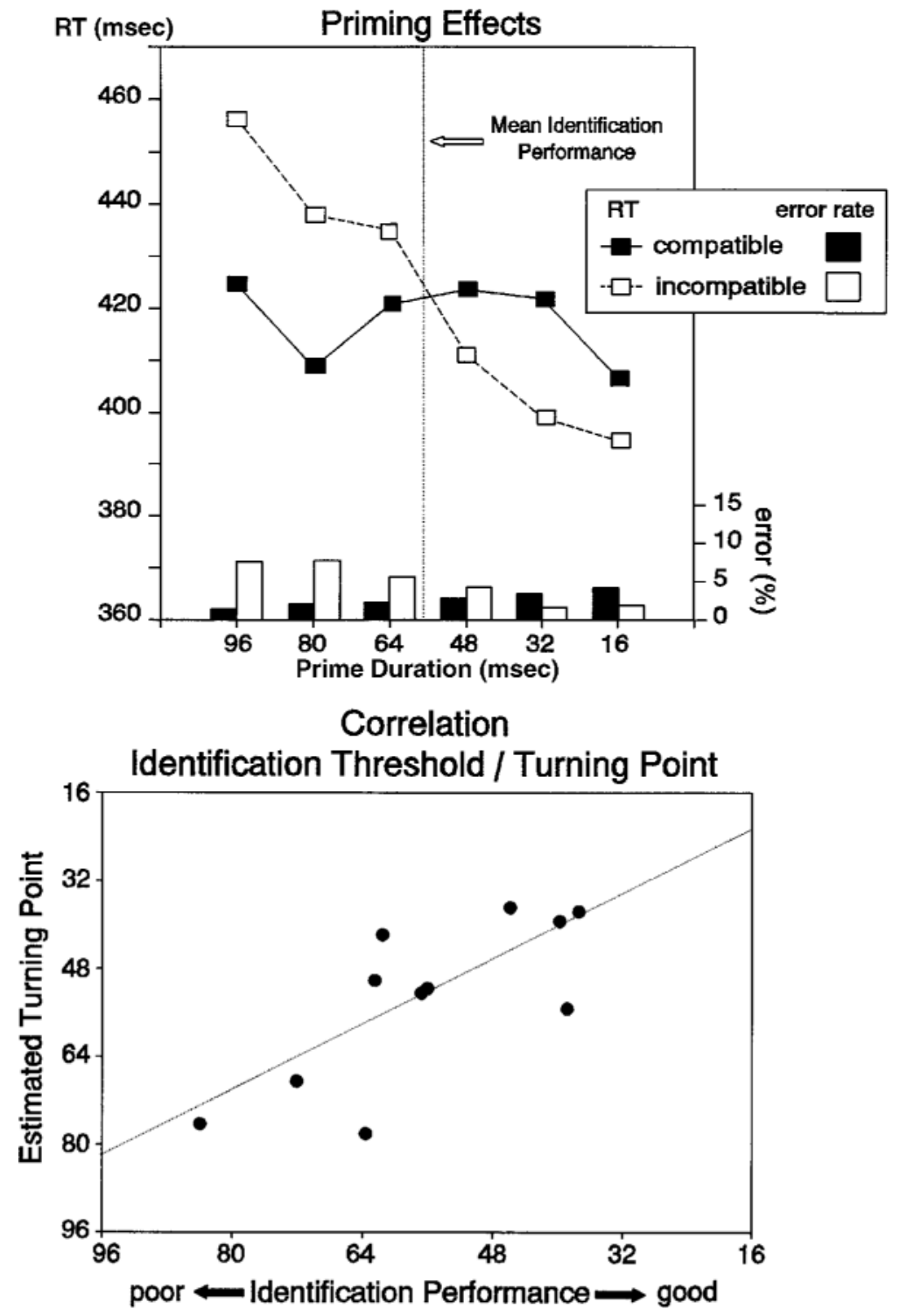

Figure 3. Results of Experiment 2. Top panel: Reaction times (line graphs) and error rates (bar graphs) in compatible trials (black) and incompatible trials (white) for different prime durations from 96 to $16 \mathrm{msec}$. The vertical line indicates the prime identification threshold along the prime visibility gradient as determined with psychophysical staircases. Bottom panel: Individual estimated turning points from positive to negative compatibility effects plotted against individual prime identification thresholds. Both values are defined in terms of prime duration (in milliseconds). Low values indicate "early" turning points and poor identification performance; high values indicate "late" turning points and good identification performance.

along a "visibility gradient," and prime identification thresholds were determined separately using psychophysical staircases. The inhibition of a primed response was inferred from negative compatibility effects (performance costs for compatible trials), while positive compatibility effects were assumed to reflect the absence of response inhibition.
The results obtained in Experiments 1 and 2 are entirely in line with the prediction that response inhibition will be found when masking prevents the conscious awareness of a prime stimulus, but not when the primes remain above the identification threshold. Positive compatibility effects were observed for suprathreshold primes, and negative compatibility effects were present when primes could not 
be consciously perceived. In Experiment 1, positive compatibility effects observed when masks were absent or contained only a few line elements turned into negative compatibility effects when mask density was increased. In Experiment 2, positive compatibility effects with longduration primes gave way to negative effects as prime duration was reduced. Averaged across participants, the point along the prime visibility gradient where positive compatibility effects turned into negative effects was numerically close to the mean identification threshold. Most importantly, significant correlations between prime identification thresholds and response inhibition onset estimates were obtained in both experiments, demonstrating that identification thresholds can predict the point along the prime visibility gradient where response inhibition affects performance. Participants with good prime identification capabilities tended to show late turning points from positive to negative compatibility effects, whereas poor identification performance was generally accompanied by early turning points.

These results suggest that the conscious awareness of a prime stimulus and the presence or absence of response inhibition in subliminal priming are linked. When perceptual representations of a prime are eliminated by a subsequent mask, these primes can no longer be identified, and evidence for response inhibition is observed. In contrast, with incomplete masking, primes remain accessible to conscious awareness (as inferred from above chance identification performance), and no response inhibition is elicited. We assume that the absence of response inhibition with suprathreshold primes is due to the fact that consciously accessible perceptual information counteracts the automatic operation of self-inhibitory motor control circuits. The presence and absence of conscious awareness and the absence or presence of response inhibition observed in this study could be parallel but independent consequences of the same underlying process (the complete or incomplete removal of a perceptual representation). Alternatively, the fact that primes are accessible (or inaccessible) to conscious awareness may be causally responsible for the absence (or presence) of response inhibition. The present findings are consistent with both interpretations.

Recent debates on subliminal perception have underlined the importance of finding qualitative differences in the effects of suprathreshold and subthreshold information. Given the notorious difficulties of unequivocally demonstrating perception without awareness (see Holender, 1986, for a critical review), the presence of such differences may be the most convincing criterion for distinguishing conscious processes from unconscious processes (Cheesman \& Merikle, 1986). Our finding that conscious awareness and response inhibition in subliminal priming are correlated provides new evidence that such qualitative differences do in fact exist. It has been argued that although automatic response tendencies can be triggered by subliminal stimuli, conscious awareness is necessary for the strategic control of intentional action (Cheesman \& Merikle, 1986; Marcel, 1980; Merikle \& Joordens, 1997). The present results suggest that consciously available in- formation is also relevant when automatic effects of selfinhibitory motor control mechanisms have to be overcome in order to initiate or maintain intentional action.

\section{REFERENCES}

Cheesman, J., \& Merikle, P. M. (1986). Distinguishing conscious from unconscious perceptual processes. Canadian Journal of Psychology, 40, 343-367.

Dehaene, S., Naccache,L., Le Clec'H, G., Koechlin, E., Mueller, M., Dehaene-Lambertz, G., van de Moortele, P.-F., \& Le Bihan, D. (1999). Imaging unconscious semantic priming. Nature, 395, 597-600.

EIMER, M. (1998). The lateralized readiness potential as an on-line measure of central response activation processes. Behavior Research Methods, Instruments, \& Computers, 30, 146-156.

EIMER, M. (1999). Facilitatory and inhibitory effects of masked prime stimuli on motor activation and behavioral performance. Acta Psychologica, 101, 293-313.

Eimer, M., \& Schlaghecken, F. (1998). Effects of masked stimuli on motor activation: Behavioral and electrophysiological evidence. Journal of Experimental Psychology: Human Perception \& Performance, 24, 1737-1747.

Holender, D. (1986). Semantic activation without conscious identification in dichotic listening, parafoveal vision, and visual masking: A survey and appraisal. Behavioral \& Brain Sciences, 9, 1-66.

Houghton, G., \& Tipper, S. P. (1994). A model of inhibitory mechanisms in selective attention. In D. Dagenbach \& T. H. Carr (Eds.), Inhibitory processes in attention, memory, and language (pp. 53-112). San Diego: Academic Press.

KaERnBACH, C. (1991). Simple adaptive testing with the weighted up-down method. Perception \& Psychophysics, 49, 227-229.

Leuthold, H., \& Kopp, B. (1998). Mechanisms of priming by masked stimuli: Inferences from event-related brain potentials. Psychological Science, 9, 263-269.

MARCEL, A. J. (1980). Conscious and preconscious recognition of polysemous words: Locating the selective effects of prior verbal context. In R. S. Nickerson (Ed.), Attention and Performance VIII (pp. 435-457). Hillsdale, NJ: Erlbaum.

Merikle, P. M., \& Joordens, S. (1997). Parallels between perception without attention and perception without awareness. Consciousness \& Cognition, 6, 219-236.

Neumann, O. (1990). Direct parameter specification and the concept of perception. Psychological Research, 52, 207-215.

Neumann, O., \& Klotz, W. (1994). Motor responses to nonreportable, masked stimuli: Where is the limit of direct parameter specification? In C. Umiltà \& M. Moskovitch (Eds.), Attention and Performance XV (pp. 123-150). Cambridge, MA: MIT Press.

Schlaghecken,F., \& Eimer, M. (1997). The influence of subliminally presented primes on response preparation. Sprache und Kognition, 16, $166-175$.

Schlaghecken, F., \& Eimer, M. (2000). A central-peripheral asymmetry in masked priming. Perception \& Psychophysics, 62, 1367-1382.

Schlaghecken, F., \& Eimer M. (2002). Motor activation with and without inhibition: Evidence for a threshold mechanism in motor control. Perception \& Psychophysics, 64, 148-162.

\section{NOTES}

1. We employed this weighted up-down method rather than the simple up-down method ( 1 up, 1 down) because of the need to keep attention focused on the discrimination task. With a simple up-down method, performance quickly converges on a 50\% accuracy (chance) level. When participants feel subjectively unable to make discriminative judgements for an extended sequence of trials, their task motivation tends to decrease, which may compromise threshold estimates.

2 . The only exception was found in the mask 20 condition, where negative compatibility effect failed to reach significance for error rates.

(Manuscript received January 25, 2001; revision accepted for publication September 19, 2001.) 